\title{
A Recursive Coupling-decoupling Approach to Improve Experimental Frequency Based Substructuring Results
}

\author{
${ }^{1,2}$ R. Cumbo, ${ }^{1}$ S. Manzato, ${ }^{2}$ G. Coppotelli, ${ }^{2}$ A. Fregolent, ${ }^{3}$ W. D’Ambrogio \\ ${ }^{1}$ Siemens Industry Software NV, Interleuvenlaan 68, 3001, Leuven, Belgium \\ ${ }^{2}$ Università degli Studi di Roma "La Sapienza", Dipartimento di Ingegneria Meccanica e \\ Aerospaziale, Via Eudossiana 18, 00184 Roma, Italy \\ ${ }^{3}$ Università degli Studi dell'Aquila, DIIE, Via G. Gronchi 18, I 67100 L'Aquila, Italy
}

\begin{abstract}
Substructure decoupling techniques allow identifying the dynamic behavior of a substructure starting from the dynamic behavior or the assembled system and a residual subsystem. Standard approaches rely on the knowledge of all FRFs at the interface DOFs between the two substructures. However, as these typically include also rotational DOFs which are extremely difficult and most of the time impossible to measure, several techniques have been investigated to overcome these limitations. A very attractive solution consists in defining mixed or pseudo interfaces, that allow to substitute unmeasurable coupling DOFs with internal DOFs on the residual substructure. Additionally, smoothing/denoising techniques have been proposed to reduce the detrimental effect of FRF noise and inconsistencies on the decoupling results. Starting from these results, some recent analysis on the possibility of combining coupling and decoupling FBS to validate the results and compensate for inconsistencies will be presented in this paper. The proposed method relies on errors introduced in the substructuring process when assuming that the interface behaves rigidly, while it is generally known that this assumption is seldom verified. Consequently, a recursive coupling-decoupling approach will be used to improve the estimation of the dynamic response of either the residual structure (for decoupling) or the assembly (for coupling). The method, validated on analytical data, will be here analyzed on a numerical example inspired by an experimental campaign used to validate the finite element models and on which standard substructuring techniques showed some limitations. The results discussed in this paper will be then used as guidelines to apply the proposed methodologies on experimental data in the future.
\end{abstract}

KEYWORDS: Substructuring, FBS, Coupling, Decoupling, Connections.

\section{INTRODUCTION}

Dynamic Substructuring (DS) is a technique allowing the componentwise analysis of a structural system. In the framework of dynamic analysis, it has some important advantages over global methods where the entire problem is handled at once [1]:

- It allows the experimental evaluation of the dynamic behavior of structures that are too large or complex to analyze as a whole (as for example an aircraft). Numerically, this holds when the number of DoFs is such that solution techniques cannot find results in a reasonable time

- $\quad$ By analyzing the subsystems, local dynamic behavior can be recognized more easily than when the entire system is analyzed

- DS also allows the identification of internal modifications referred to a subsystem

The concept of substructuring holds its roots in the field of domain decomposition and includes two main branches: decoupling and coupling [2]. Given a known assembled system RU, decoupling consists in the identification of the dynamics of the unknown structure $U$ by decoupling R (residual substructure) from RU. Coupling, on the other hand, evaluates the dynamic behavior of an unknown RU system starting from the knowledge of the two subsystems $\mathrm{R}$ and U. Even if structural modification techniques are generally not considered as substructuring, the two concepts 
are instead almost the same [3]: one could see the coupling problem as the structural modification of $\mathrm{R}$ caused by the addition of subsystem U. Same considerations can be done for the decoupling: the subtraction of R is a negative modification of mass, stiffness and damping of the assembled system RU [4]. DS can be studied in three different domains: physical, modal or frequency. The latter is known as Frequency Based Substructuring (FBS) and it is the one analyzed and applied in this paper. As already explained, the DS can be applied both numerically and experimentally, or even to combine numerical and experimental substructure models. The main issues in this field, extensively reported in literature, are related to the applicability of the technique to experimental data. At first, the intrinsic formulation of the problem together with the presence of noise will result in an ill-conditioned matrix which has to be inverted in the substructuring formulation (see for instance [5], [6]). The second problem is linked to the actual measurement process: sometimes it is really difficult to compensate for the impossibility of measuring the rotational DoFs, That leads to the definition of incorrect or incomplete compatibility and equilibrium equations imposed at the interface, especially for the coupling approach [7], [8], [9].

After a brief theoretical summary of the FBS, different developed methods will be explained. They are focused on the identification of the connection errors that usually lead to wrong results in the experimental measurements. The aim of the error identification at the connection is to find an useful way to apply this information in next substructuring applications of the same system.

\section{FRAMEWORK FOR FBS}

Considering a generic linear and time-invariant subsystem $\mathrm{R}$, the equation of motion in the physical domain is:

$$
Z^{R}(\omega) u^{R}(\omega)=f^{R}(\omega)+g^{R}(\omega)
$$

where $Z^{R}, u^{R}, f^{R}, g^{R}$ are respectively the dynamic stiffness matrix, the vector of DoFs, the external force vector and the vector of connecting forces with the other substructures. The same equation can be written also for the unknown substructure U. To evaluate the coupled dynamic response of the two substructures, the compatibility and the equilibrium equations at the connection points must be added to the resulting equations of motion of the two subsystems. The former condition implies:

$$
u_{c}^{R}-u_{c}^{U}=0
$$

This condition can be generally expressed as $B u=0$, where each row of B corresponds to a pair of matching DoFs. Note that the Eq.(2) is referred to a rigid connection. The equilibrium condition implies that the sum of the connecting forces across a connection for a pair of matching DoFs is zero:

$$
g_{c}^{R}+g_{c}^{U}=0
$$

For the internal DoFs of each subsystem $g=0$ holds. For generalization, the above conditions can be expressed as $L^{T} g=0$, where the matrix $\mathrm{L}$ is the Boolean localization matrix and it is defined as the nullspace of $\mathrm{B}$. The final system of equations will be:

$$
\left\{\begin{array}{c}
Z(\omega) u(\omega)=f(\omega)+g(\omega) \\
B u(\omega)=0 \\
L^{T} g(\omega)=0
\end{array}\right.
$$

In which $u(\omega)$ is a vector with all components of the DoFs of $\mathrm{R}$ and $\mathrm{U}$ and $Z(\omega)$ is the following diagonal matrix:

$$
Z(\omega)=\left[\begin{array}{cc}
Z^{R}(\omega) & 0 \\
0 & Z^{U}(\omega)
\end{array}\right]
$$

The system of Eq.(4) represents the so-called 3-field formulation. Starting from the 3-field formulation, several assembly techniques can be devised: dual, primal and hybrid assembly (see [10]). Even if all assembly techniques give the same results for $N_{E}=N_{C}$ (i.e. the number of compatibility DoFs is the same as the number of equilibrium DoFs), the dual assembly involves the least number of matrix inversions and for this reason it will be used in the following sections. In the dual assembly, the equilibrium condition $L^{T} g(\omega)=0$ is satisfied by writing $g=-B^{T} \lambda$. Eq.(4) becomes: 


$$
\left\{\begin{array}{c}
Z(\omega) u(\omega)+B^{T} \lambda=f(\omega) \\
B u(\omega)=0
\end{array}\right.
$$

\subsection{FBS COUPLING}

Considering the two substructures, let us indicate the internal DoFs of $\mathrm{R}$ and $\mathrm{U}$ respectively with $r$ and $u$ and the connection DoFs with $c$. The algorithm proposed by Jetmundsen provides less matrix inversions, thus overcoming a big amount of numerical problems:

$$
H^{R U}=\left[\begin{array}{ccc}
H_{r r}^{R} & H_{r c}^{R} & 0 \\
H_{c r}^{R} & H_{c c}^{R} & 0 \\
0 & 0 & H_{u u}^{U}
\end{array}\right]-\left[\begin{array}{c}
H_{r c}^{R} \\
H_{c c}^{R} \\
-H_{u c}^{U}
\end{array}\right]\left[H_{c c}^{R}+H_{c c}^{U}\right]^{-1}\left[\begin{array}{c}
H_{r c}^{R} \\
H_{c c}^{R} \\
-H_{u c}^{U}
\end{array}\right]^{T}
$$

In Eq.(7) and in the following, the dependency of the Frequency Response Function to the frequency will be omitted for simplicity. It's worth to note that in the latter equation the connecting DoFs are assumed to belong to R. If one assumes these DoFs belonging to $\mathrm{U}$, the coupled equation is modified as:

$$
H^{R U}=\left[\begin{array}{ccc}
H_{r r}^{R} & 0 & 0 \\
0 & H_{c c}^{U} & H_{c u}^{U} \\
0 & H_{u c}^{U} & H_{u u}^{U}
\end{array}\right]-\left[\begin{array}{c}
-H_{r c}^{R} \\
H_{c c}^{U} \\
H_{u c}^{U}
\end{array}\right]\left[H_{c c}^{R}+H_{c c}^{U}\right]^{-1}\left[\begin{array}{c}
-H_{r c}^{R} \\
H_{c c}^{R} \\
H_{u c}^{U}
\end{array}\right]^{T}
$$

However, both Eq.(7) and (8) will lead to the same results [11]. Using numerical data, these two equations lead to the same FRF matrix of RU. Using experimental data only, on the other hand, the FRFs referred to coupling DoFs are slightly different depending on the equation being used.

\subsection{FBS DECOUPLING}

The decoupling problem aims at finding the dynamic behavior of the unknown $U$ substructure, starting from the knowledge of RU and R. In general U is influenced not only by external forces but also by connection force of R. Hence, in order to decouple, it is needed to apply to RU additional force opposing these connection forces: the effect of this calculation is thus the behavior of the substructure $U$ without the influence of $R$. Starting from the dual formulation approach:

$$
H^{U}=\left(H^{R U}-H^{R U} B_{R U}^{T}\left(B_{R U} H^{R U} B_{R U}^{T}-B_{R} H^{R} B_{R}^{T}\right)^{-1} B_{R U} H^{R U}\right)
$$

Note that the above equation is valid in case of collocated interface and it is the one that we will use in this paper. If one wants to use the non-collocated interface, it's needed to define different Boolean matrices for equilibrium and compatibility conditions ([10]). In order to build the Boolean matrix in the decoupling problem, the type of interface has to be chosen among those displayed in Fig. 1.

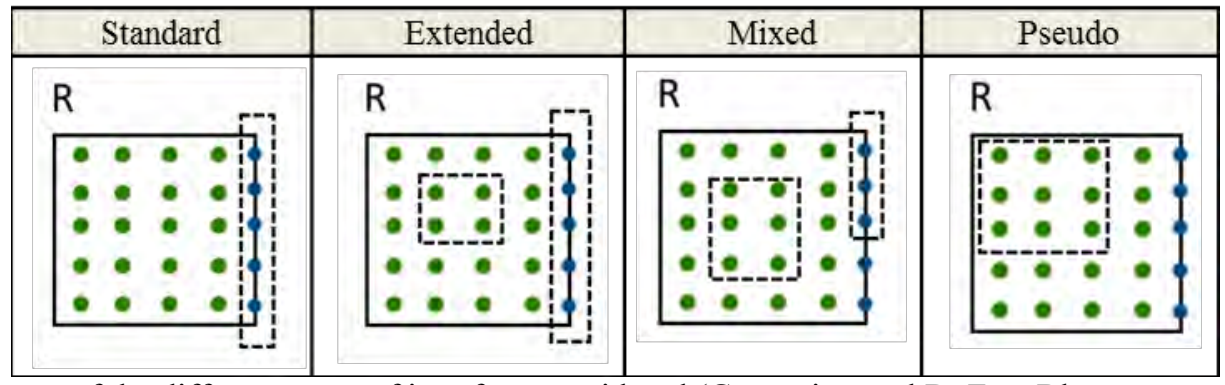

Fig. 1: Scheme of the different types of interface considered (Green: internal DoFs - Blue: connection DoFs).

Experimentally, there are many difficulties in measuring the connection DoFs of the assembled structure RU. This means that it is generally not possible to use the first three types of interface displayed in Fig. 1. However, when using the pseudo-interface, a right choice of the internal DoFs is needed: in fact, in order to perform a good decoupling of $\mathrm{R}$ from $\mathrm{RU}$, it is important to measure all the information about the dynamics of $\mathrm{R}$ in the considered range of frequency. 


\section{ITERATIVE APPROACHES ON DYNAMIC SUBSTRUCTURING}

The two iterative approaches that will be explained in this section were developed with the aim of finding the connection error resulting from DS application with experimental data. Information about identified connection error can be then used again in future DS applications on the same structures :

- During the design phase of a project, one might want to modify one of the subsystems and verify its effect on the dynamics of the assembly. A useful way of analyzing how the complete system changes is by studying it experimentally. Considering the coupling problem, one might be able to compensate for connection errors/uncertainties using a priori information. This connection compensation value was previously found by applying one of the proposed iterative approaches. In this way, the second measurement of the complete system RU is not needed anymore (Fig. 2).

- $\quad$ Another example is given in Fig. 3:

- First Step: the dynamic response of each structure (R, $\mathrm{U}$ and $\mathrm{RU}$ ) is known at time $T_{0}$. Starting from this, one of the iterative approaches to find the connection error can be applied to improve the quality of the coupling results

- Second Step: after the operative life (at time $\bar{T}$ ), the subsystem U (i.e. the engine) is subject to dynamic modification or needs to be updated with a newer model. Assuming no changing on R, the connection error can be applied on the coupled structure in order to reliably evaluate the dynamic response based on the dynamic model of the new $\mathrm{U}$.

DESIGN 1

\section{DESIGN 2}

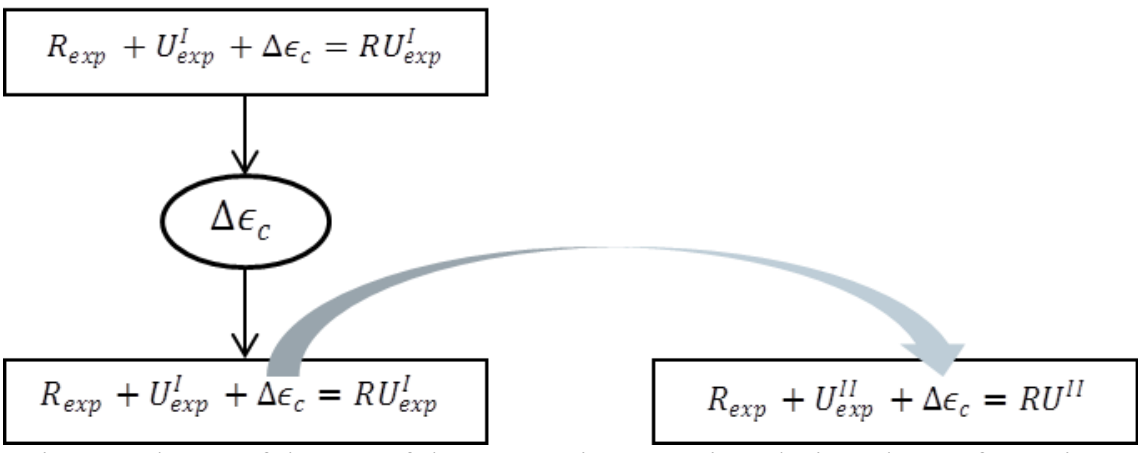

Fig. 2: Scheme of the use of the connection error in a design phase of a project.
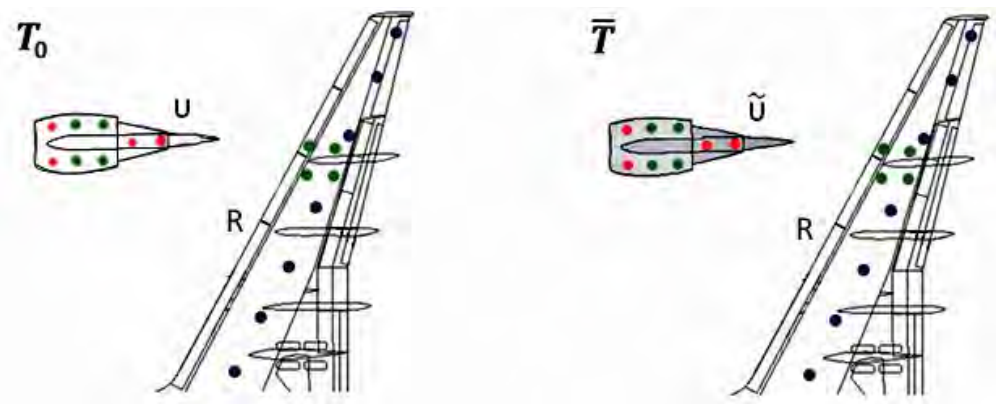

Fig. 3: Substructure U (engine) subject to a modification at a certain time $\bar{T}$ - Substructure R: wing.

In the following section, the proposed approaches will be explained: FCU-R (Frequency Connection Updating Rigid) and FCU-F (Frequency Connection Updating - Flexible). The method to be applied on each specific case will depend on the assumption made to model the connection; in particular, when introducing the method, we will refere to rigid or flexible connections (depending on the compatibility condition applied) and point vs. multipoint connections, which depend on the size of the connection area. 
3.1 FCU-R: Frequency Connection Updating - Rigid

The first discussed method is based on the application of the Predictor-Corrector (PC) Frequency Domain Updating Method ([12], [13]) on DS. The basic idea is to update the dynamic measured RU model in order to find the uncertainty in terms of mass and stiffness at the interface DoFs, such that $R+U+\Delta \epsilon_{\mathrm{c}}=R U$. It means that this procedure could represent the connection errors as a local perturbation of mass and stiffness properties $\left(\Delta \epsilon_{c}=\Delta m\right.$, $\Delta \epsilon_{c}=\Delta k$ or a combination of them) at the same local point. Rigid connections are assumed.

For this purpose, two approaches were initially investigated:

- Correlation between the exact (measured) $U$ and the decoupled one

- Correlation between the exact (measured) RU and the coupled one

The solution to the first problem will not be discussed here because it finds difficult experimental applications. A hard requirement for both techniques is that the physical mass and stiffness matrices are needed in order to apply the PC. Consequently, the FE model of each assembly has to be updated and so validated FE model are required. It is worth to note that, assuming there is no noise in the data, the error will only be associated to connection uncertainties. Furthermore, these methods are applicable only with point connections. Indeed, starting from the FE model, we locally update the FRF only at the connection points which will be subjected to the compatibility and the equilibrium equations. In case of distributed contact between the substructures, and assuming a point connection, the updating of the local properties will not allow to compensate for the lack of information. In this case one should find the minimum number of point connections which is able to represent accurately the actual contact at least in the frequency range of interest. Another problem is related to the rotational DoFs at the connection, which are typically impossible to measure experimentally. As the proposed method will not be able to compensate for them, an alternative approach is try to impose compatibility on a higher number of translation DoFs over the same contact surface. (see Sec.4.1.).

The aim of the FCU-R method is to correlate directly the measured structure RU with the one that in the substructure decoupling algorithm can give the exact $\mathrm{U}$. This can be performed in the following way:

- $\quad$ supposing that $\mathrm{U}$ and $\mathrm{R}$ are known experimentally, one can evaluate the coupled $R U_{\text {coup }}$ using the solution proposed by Jetmundsen, considering that the connection DoFs belong to the substructure U (Eq.(8))

- coming back to the decoupling problem, we can evaluate $R U_{\text {coup }}-R$ using a pseudo-interface and the results will give us the exact dynamic behavior of $U$

Therefore, we can affirm that: the coupled structure $R U_{\text {coup }}$, evaluated with the Jetmundsen equation (Eq.(8)), is such that if one decouples it from $R$ imposing a pseudo-interface, the results give the exact solution $U$.

The FCU-R can be demonstrated imposing the following system:

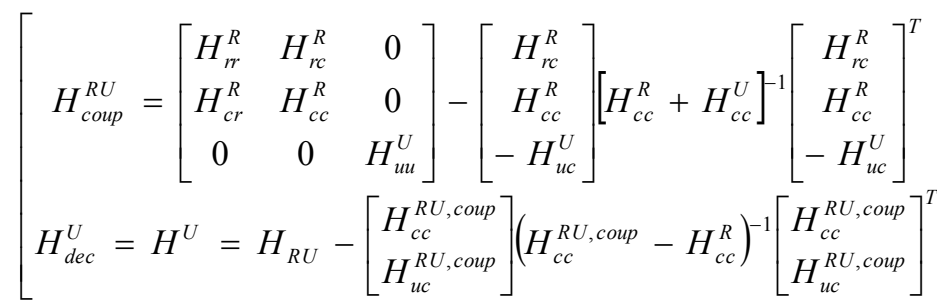

Then RU and $R U_{\text {coup }}$ are correlated using the classical PC method, updating mass and stiffness parameters only on the connection measured DoFs. Note that this method can be used also in the decoupling problem: in fact if one find the connection error, then it can be applied in a second measurement on RU. Thus, decoupling R from the modified assembled structure, one will find exactly the dynamic behavior of $U$.

\subsection{FCU-F: Frequency Correction Updating - Flexible}

This method finds applicability in those cases where there is the possibility to measure the connection DoFs of the coupled structure on both sides: the one belonging to $\mathrm{U}$ as well as the one belonging to $\mathrm{R}$. A typical example is that of two substructures connected by a spring with sufficient free length: in this case one can measure both connected points of the spring and the coupled structure RU will have one more measured DoF compared to the rigid interface. FCU-F was developed starting from the concept of compliant interface studied by De Klerk [14], where, considering no damping at the connection, the compatibility equation and the Lagrange multiplier can be written respectively as:

$$
B u=\Delta u_{c}
$$




$$
\lambda=K_{c} \Delta u_{c}=Z_{c} \Delta u_{c}
$$

The final modified expression for decoupling is then:

$$
H^{U}=\left(H^{R U}-H^{R U} B_{R U}^{T}\left(B_{R U} H^{R U} B_{R U}^{T}-B_{R} H^{R} B_{R}^{T}+H_{C}\right)^{-1} B_{R U} H^{R U}\right)
$$

with $H_{c}=Z_{c}^{-1}$. Clearly for rigid connection, $H_{c}$ goes to zero and one can find back Eq.(9).

Starting from the approach developed by De Klerk, the innovation with the FCU-R is its application in Experimental Dynamic FBS, as we are going to demonstrate in Sec.4.2 by a numerical example. Starting from the knowledge of all systems $\mathrm{R}, \mathrm{U}$ and $\mathrm{RU}$, the term $H_{c}$ can be derived as:

$$
H_{C}=-\left\{\left(\left[\begin{array}{c}
-H_{r c, C}^{R} \\
H_{u c, C}^{U}
\end{array}\right]^{T}\left[\begin{array}{c}
-H_{r c, C}^{R} \\
H_{u c, C}^{U}
\end{array}\right]\right)^{-1}\left[\begin{array}{c}
-H_{r c, C}^{R} \\
H_{u c, C}^{U}
\end{array}\right]^{T}\left(H_{r e f}^{R U}-\left[\begin{array}{cc}
H^{R} & 0 \\
0 & H^{U}
\end{array}\right]\right)\left[\begin{array}{c}
-H_{r c, C}^{R} \\
H_{u c, C}^{U}
\end{array}\right]^{T}\left(\left[\begin{array}{c}
-H_{r c, C}^{R} \\
H_{u c, C}^{U}
\end{array}\right]^{T}\left[\begin{array}{c}
-H_{r c, C}^{R} \\
H_{u c, C}^{U}
\end{array}\right]\right)^{-1}\right\}^{-1}
$$

Inverting the expression of $H_{c}$, one can find a diagonal matrix $K_{c}$ of dimensions $N_{C} x N_{C}$ in which each element will represent the value of the stiffness connection relative to that interface DoF. As this represents a physical stiffness its value will be constant at each spectral line. Both numerically and experimentally (as we will explain later) the results are not really constant because of respectively numerical errors and noise problems, or a combination of both. For both cases an averaging of the results in frequency is performed:

- $\quad$ by first smoothing the resulting frequency-dependent elements of matrix $K_{c}$, to regularize the results and cancel the effect of numerical noise

- by calculating then the mean value across frequency for each element of the matrix

Then, an iterative process of coupling-decoupling is proposed in order to enforce the regularization of the results.

Note that in case of flexible connection, the PC updating shows a hard applicability. In fact one could find the stiffness parameters by updating the coupled rigid system and comparing the latter with the reference one (with the "spring" connection) but of course the two systems will not only show a shift in frequency but also differences in modes.

\section{NUMERICAL VALIDATION}

In this section, the applicability and validation of FCU-R and FCU-F on the coupling of two beams are presented. Firstly, the analyzed system is described (Fig. 4): the substructures (horizontal beam R and vertical beam U) are coupled at four locations, which are realized by bolts (point connections). The size of the cross section is $0.8 \mathrm{~cm} \times 4 \mathrm{~cm}$. Both of them are in Aluminium and the analyzed frequency range is [0-3200] Hz. In Table 1, the natural frequencies (bending - ' $z$ ' direction) of the validated FE model of the reference structure RU and of the coupled one are reported. Note that the latter is the result of coupling between the validated FE models of $\mathrm{R}$ and $\mathrm{U}$. The difference between the reference and the coupled models are in the connection area. The FE model validation of the reference system (which was in fact experimentally analyzed - Fig. 5) led to modeling the connection points with a total of 8 spiders (4 for each substructure) around the connection points and 4 RBEs between corresponding connection points on the two substructures. We demonstrated numerically that it is possible to obtain good results on the coupling between $\mathrm{R}$ and $\mathrm{U}$ by simply applying 4 rigid connections along translational ' $\mathrm{z}$ ' DoFs (Fig. 6). Thus, by simulating the experimental coupled structure, the FE model has only 4 RBE elements. Note that it is only a simulation. In fact, experimentally, the connection area wasn't wide enough to allow measuring directly the 4 connection points with accelerometers (Fig. 5). 


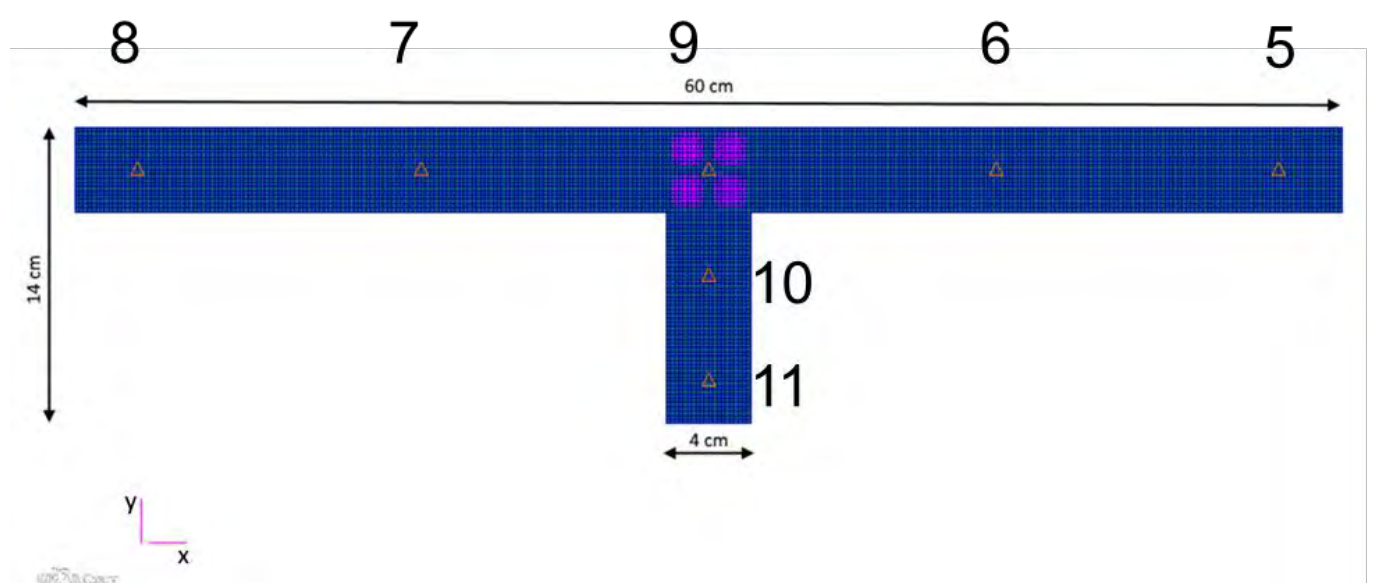

Fig. 4: Reference structure RU with name of the measured DoFs. Connection modeled by 8 spiders and 4 rigid connections on all DoFs
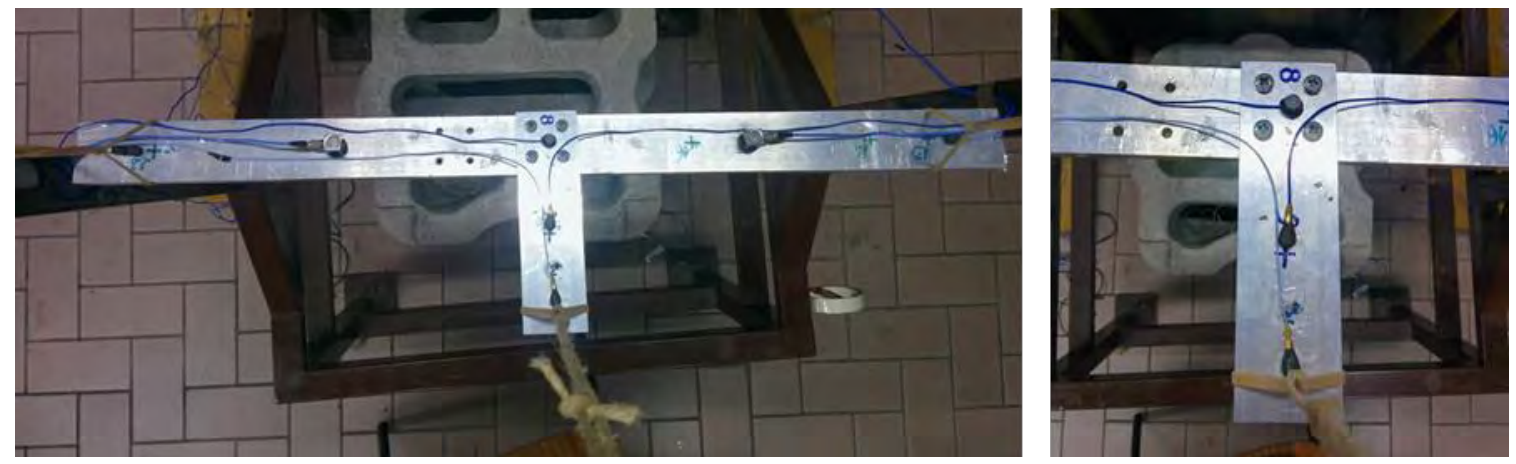

Fig. 5: Experimental setup of RU. Connection area on the right size
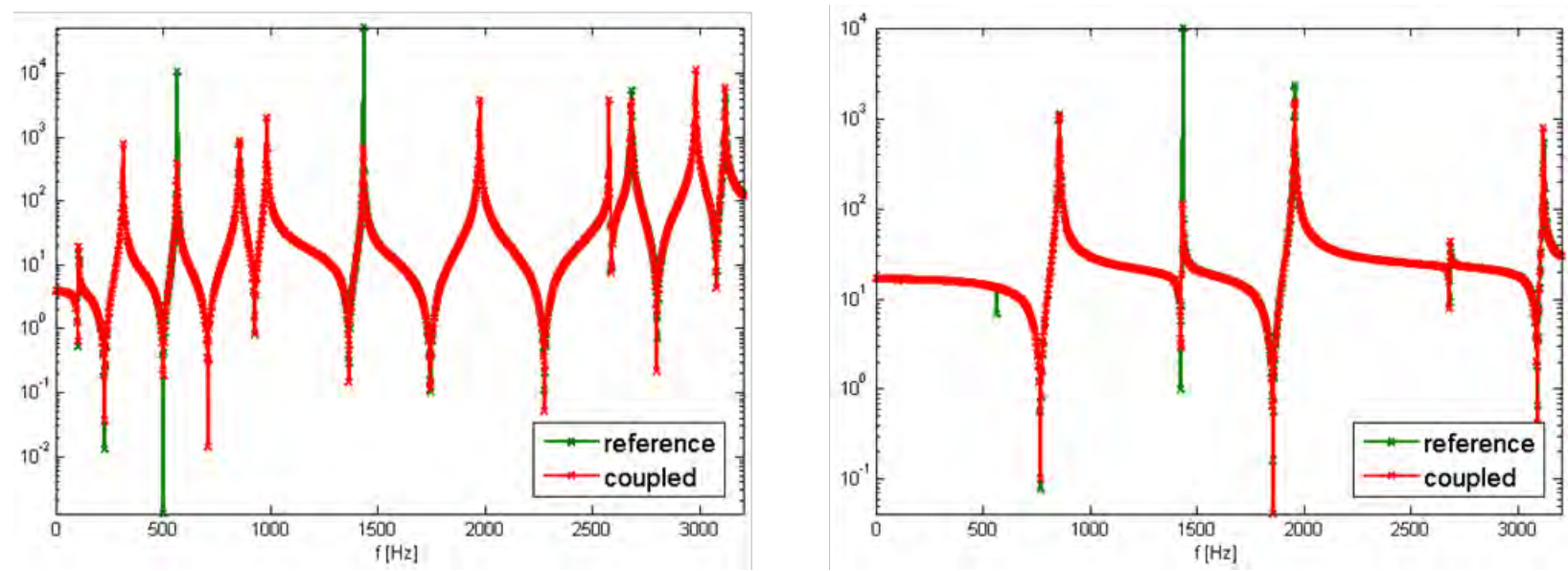

Fig. 6: FBS coupling, DoFs 7 (left) and 10 (right): comparison between FRFs of the reference case ( 8 spiders, 4 RBE all DoFs) and the simulated experimental coupling case (4 RBE ' $z$ ' DoF) 
Table 1: Natural frequencies (bending out of plane in z direction) of the coupled and the reference model RU

(Green: torsion of R and bending of U; Orange: bending of R and torsion of U; Cyan: out-of-band mode)

\begin{tabular}{ccc}
\hline Coupled Ru [Hz] & Reference RU [Hz] & Relative Error [\%] \\
\hline 107.16 & 112.60 & 4.83 \\
311.92 & 312.20 & 0.09 \\
565.66 & 591.90 & 4.43 \\
854.78 & 915.30 & 6.61 \\
1014.70 & 1019.00 & 0.42 \\
1434.40 & 1499.70 & 4.35 \\
1955.20 & 1975.90 & 1.05 \\
1974.30 & 2023.40 & 2.43 \\
2682.20 & 2808.00 & 4.48 \\
3117.50 & & \\
\hline
\end{tabular}

In Table 1, the modes showing coupling between torsion and bending are underlined. Note that these modes are very sensitive to the correct modeling of the connection: if one aims at predicting this behavior neglecting the rotational DoFs, at least all 4 connection points are required. A comparison between the reference model and a simulated experimental case (in which, because of the size of the interface area, one could measure only one connection point) is reported: in Fig. 7 and Fig. 8 the coupled modes and a result of the FBS coupling for this case (numerical data) are respectively shown. Because of the wrong assumptions at the connection, the coupling between the substructures leads to erroneous results.

Taking into account the reference model and the coupled one with 4 connections, the FCU-R and FCU-F methods will be applied and validated numerically in the following sections.

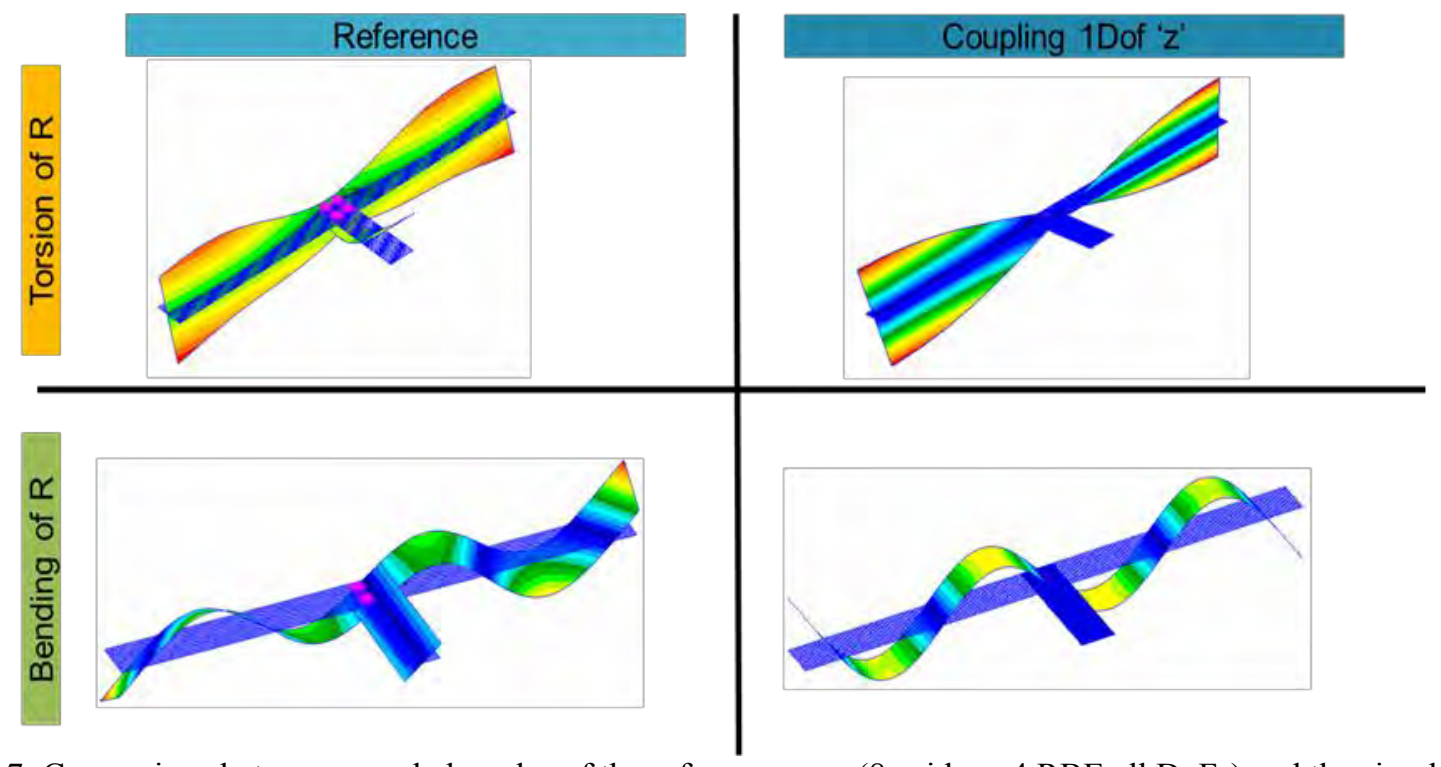

Fig. 7: Comparison between coupled modes of the reference case ( 8 spiders, 4 RBE all DoFs) and the simulated experimental coupling case (1 RBE ' $\mathrm{z}$ ' DoF) 

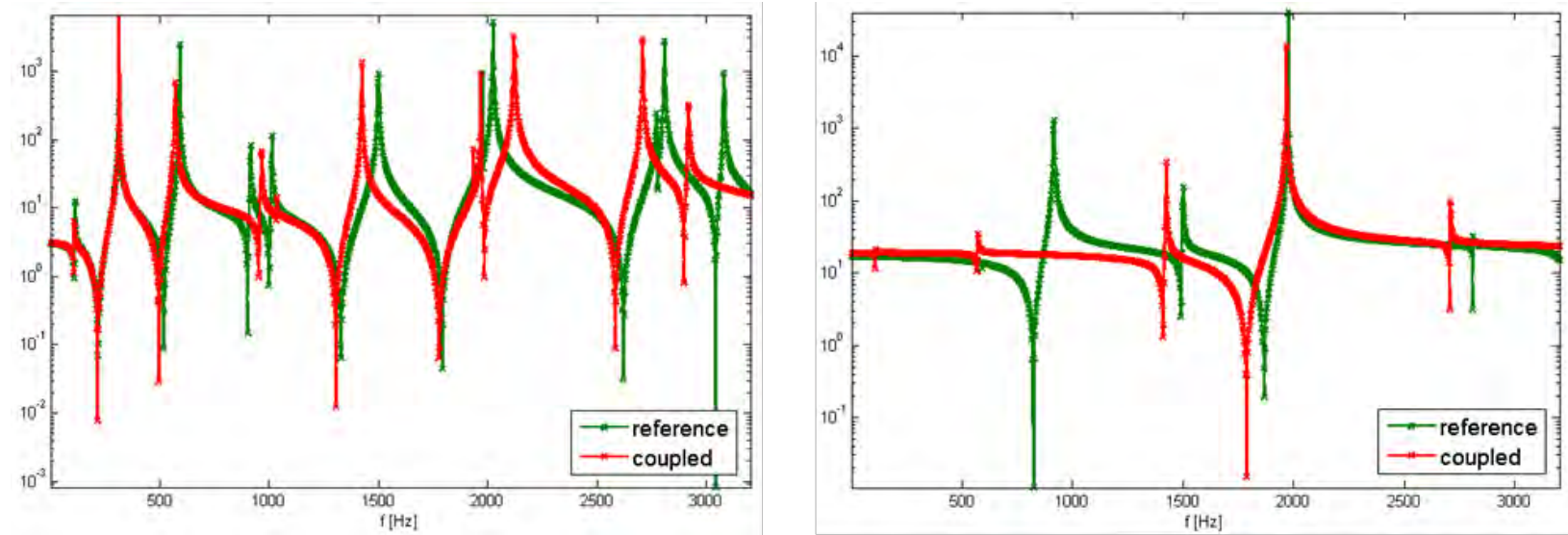

Fig. 8: FBS coupling, DoFs 7 (left) and 10 (right): comparison between FRFs of the reference case ( 8 spiders, 4

RBE all DoFs) and the simulated experimental coupling case (1 RBE ' $z$ ' DoF)

\subsection{APPLICATION OF FCU-R}

In this section, we will validate the applicability of FCU-R using the system discussed in the previous paragraph. To achieve it, a first analysis was performed to find a distributed parameters error: material properties of the connection area $\left(E_{c}\right.$ and $\left.\rho_{c}\right)$ were found as a result of an optimization analysis in Siemens LMS Virtual.Lab (Fig. 9). It should be noted that, to increase the efficiency of the calculations, a Craig-Bampton model order reduction was applied on both substructures before coupling. Although the accuracy of the reduction was verified, some small errors were still present leading to a slight decrease in the correlation with the reference coupled model shown in Table 1

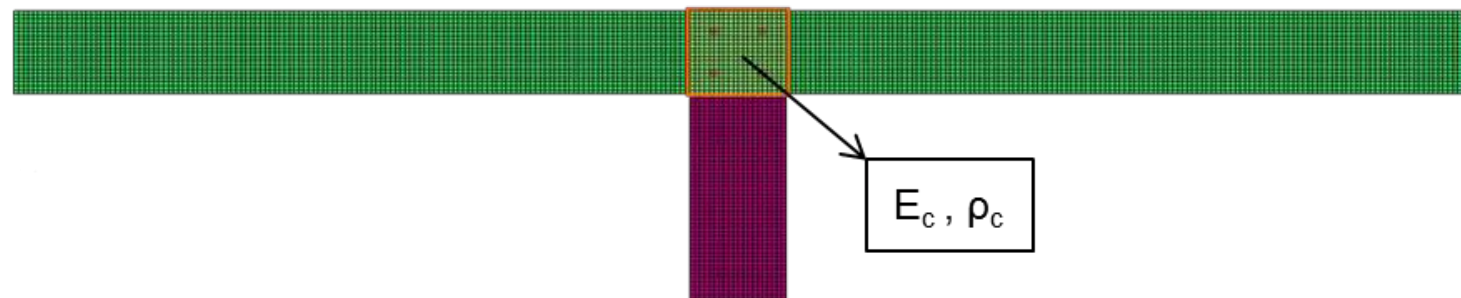

Fig. 9: Definition of different material properties of the connection area.

The obtained optimal values are: $E_{c}=90 G P a$ and $\rho_{c}=2400 \mathrm{~kg} / \mathrm{m}^{3}$. The percentage errors before and after the update are reported in Fig. 10. As one can note, they are reduced for all frequencies, in particular in the mediumhigh range where the dynamics of the connection appears.

To simulate a modification of the substructure $\mathrm{U}$ at the time $\bar{T}$, a lumped mass of about $0.006 \mathrm{~kg}$ was added at the center of the substructure $U$. Then, the previously obtained connection property values $E_{c}$ and $\rho_{c}$ are applied on the coupled system. The error percentage before and after the update is reported in Fig. 11 and as one can note, its reduction is very similar to the one obtained with the unmodified system. Thus the connection error can be represented in terms of mass and stiffness parameters.

Now, the analysis is repeated in terms of lumped parameters at the connection DoFs (4 for the analyzed system). In order to do that, the input parameters are set as variation of the nodal mass at the 4 connection points (same values). Simulating an experimental case, the two models were correlated on 10 "measured" points: 4 internal of R, 2 internal of U, 4 connections. The initial and final correlations are shown in Fig. 12: the updating focused on a narrower frequency range $([0-700] \mathrm{Hz})$ as at higher frequency the correlation decreases and a low correlation will negatively affect the applicability of the PC method. In Fig. 12, the shape and amplitude correlation coefficients [12] (respectively $\chi_{s}, \chi_{a}$ ) show that, after applying the obtained mass parameter, the first three natural frequencies (those in the analyzed range) are almost perfectly correlated. Note that the peaks of the green curve imply that the amplitude at the natural frequencies are not really the same, but anyway the correlation is acceptable. Furthermore these peaks can be explained by the absence of damping in the FE model and by numerical errors. The obtained variation of nodal mass is about $-17.5 \%$ for each connection point. The negative value is due to the fact that the coupled model has lower natural frequencies than the reference one and consequently the higher stiffness of the latter has to be compensated. 


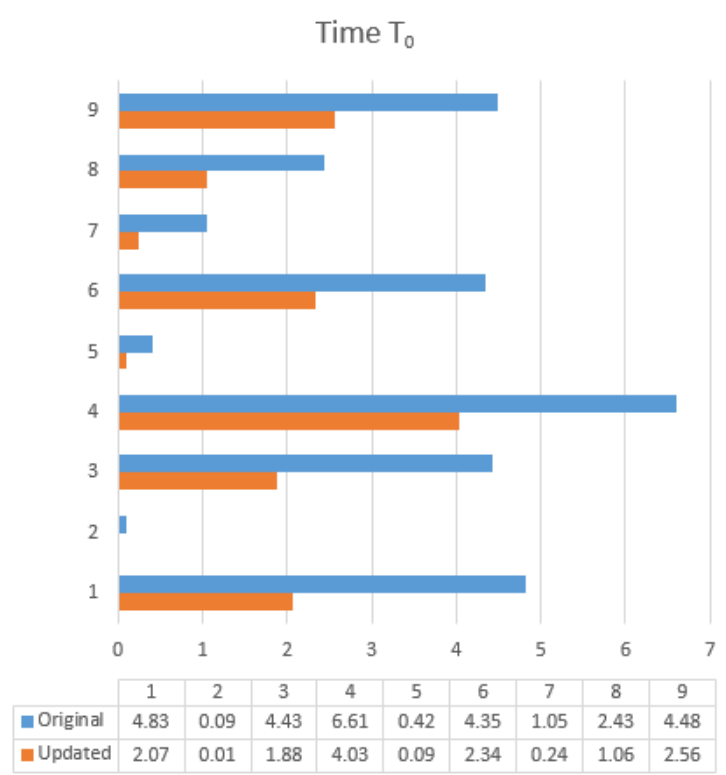

Fig. 10: Relative frequencies errors (x-axis) for each natural mode (y-axis) between coupled and reference model at time $T_{0}$ before (Blue) and after (orange) updating.

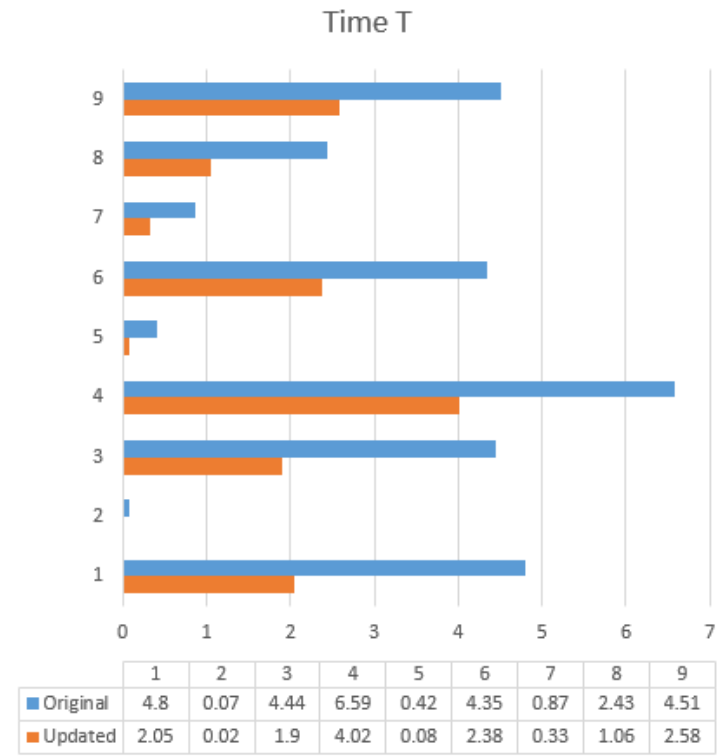

Fig. 11: Relative frequencies errors (x-axis) for each natural mode (y-axis) between coupled and reference model at time $\bar{T}$ before (Blue) and after (red) updating.
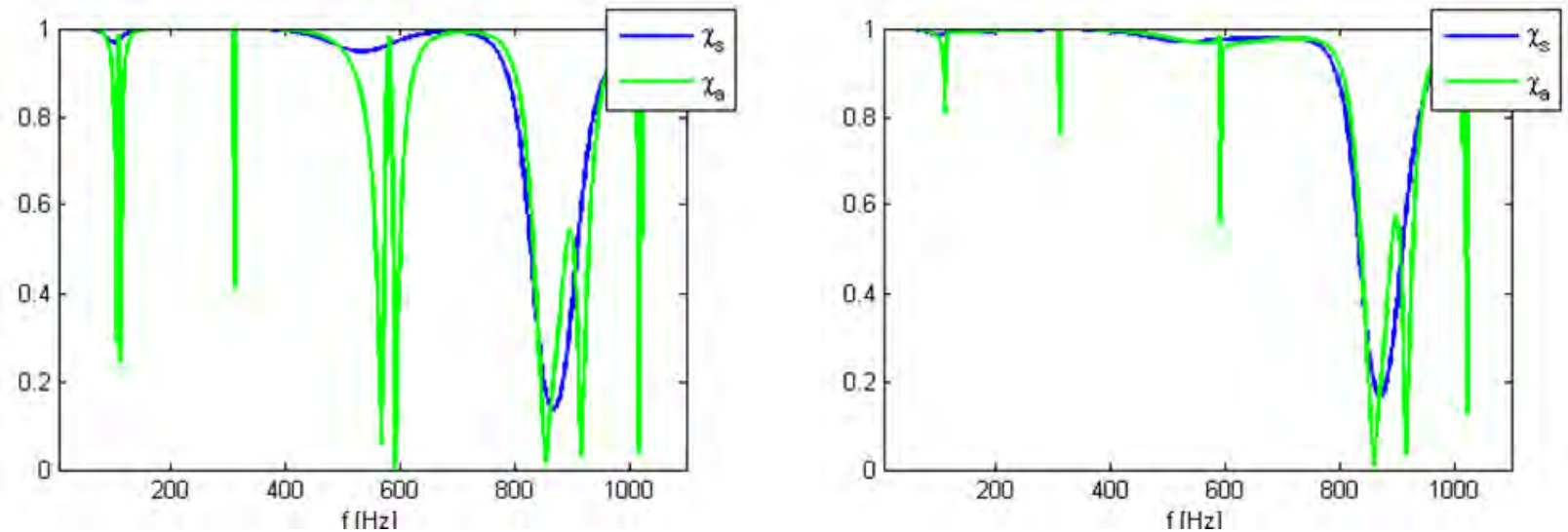

Fig. 12: Correlation between the reference and the coupled systems (time $T_{0}$ ) before (left) and after (right) updating - value of the obtained mass error: $-17.5 \%$ of the nodal mass of each connection DoFs.

The resulting mass error at the connection was then applied on the previously simulated modified system at time $\bar{T}$ (Fig. 13): the effect is almost the same and the results can be considered very good in the analyzed frequency range. Note that if, instead of a concentrated mass, one would set the mass and stiffness properties of the connection points as input for the PC method, the procedure will be practically the same as the one performed in Virtual.Lab: in fact the PC method will update the element mass and stiffness matrices around the location of the connection DoFs. 

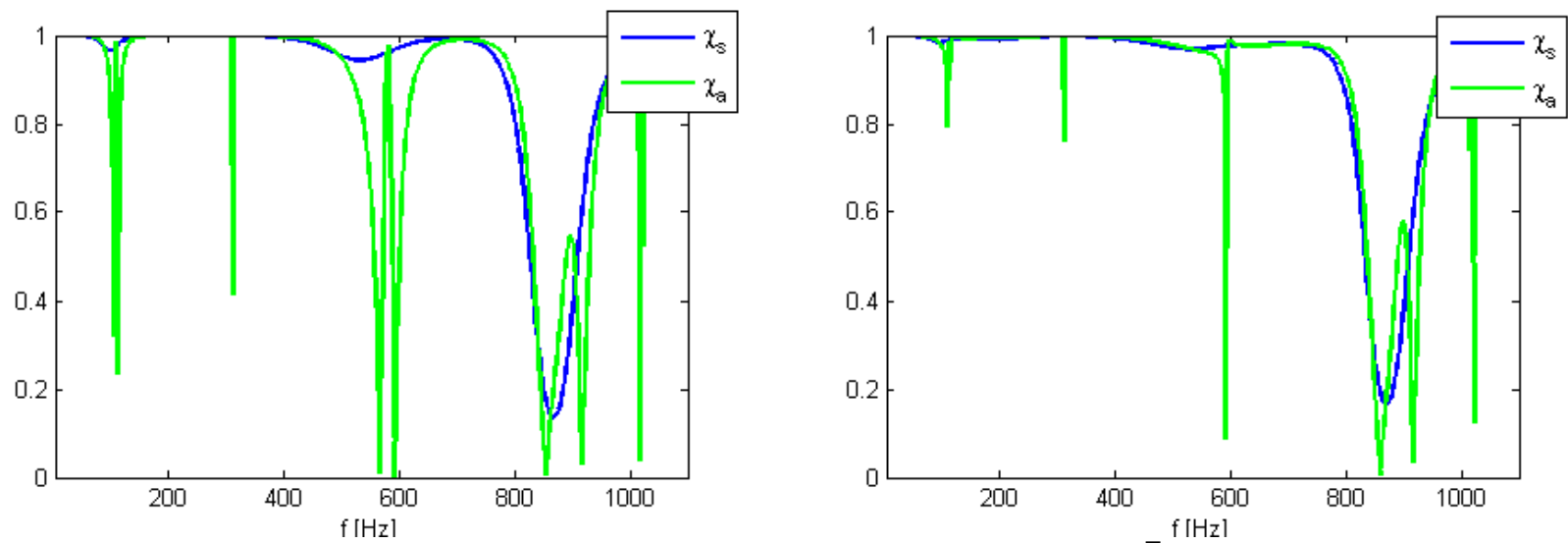

Fig. 13: Correlation between the reference and the coupled modified systems (time $\bar{T}$ ) before (left) and after (center) the application of the previously obtained mass connection error.

\subsection{APPLICATION OF FCU-F}

As discussed in Section 3.2, this method can find applicability in those cases where it is possible to measure at both sides of the connection DoFs in $\mathrm{R}$ and in $\mathrm{U}$. In fact, considering 4 springs as shown in Fig.14, the connection DoFs of $\mathrm{R}$ and its $\mathrm{U}$ counterpart are not coincident as it happened for a rigid connection. The same system of beams presented in the previous section will be considered to apply the FCU-F. Four flexible connections between the substructures are defined in the FE model. Also some noise will be added to simulate an experimental case.

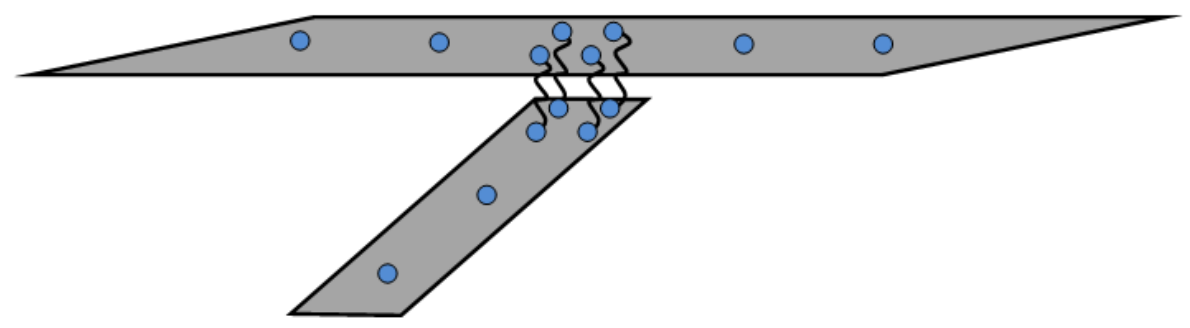

Fig. 14: Four flexible connections between R (8 “measured" points) and U (6 “measured" points).

The following steps are performed:

- Evaluate the FRF matrices in ' $z$ ' direction of R [8x8], U [6x6] and RU [14x14]. The latter, considered as the reference assembled structure, was made by connecting $\mathrm{R}$ and $\mathrm{U}$ with 4 springs with equal stiffness value $2 \cdot 10^{10} \mathrm{~N} / \mathrm{m}$

- Evaluate Eq.(14) leading to a matrix with dimension [4x4]. By inverting it, a diagonal matrix is obtained with the values of the imposed connection stiffnesses.

In case of noisy data, additional steps are needed:

- $\quad 0.001 \%$ of complex circular noise is added to the simulated FRFs

- $\quad$ Elements of $H_{c}^{-1}$ matrix are smoothed

- The average frequency value of each element on the diagonal of $H_{c}^{-1}$ is calcualted

- Reciprocity is imposed on $K_{c}$ (lower triangular matrix equal to the upper one) in order to allow the diagonalization of the matrix (in fact, for noisy data, the matrix $K_{c}$ is not diagonal)

- The complex $\mathrm{K}_{\mathrm{c}}$ matrix is realized using the following approach:

$$
k_{\text {real }}=\operatorname{Re}\left(k_{\text {im }}\right)+\operatorname{Im}\left(k_{\text {im }}\right) \cdot\left(\operatorname{Re}\left(k_{\text {im }}\right)^{\prime} \cdot \operatorname{Re}\left(k_{\text {im }}\right)\right)^{-1} \cdot \operatorname{Re}\left(k_{\text {im }}\right)^{\prime} \cdot \operatorname{Im}\left(k_{\text {im }}\right)
$$

- The mean value between the obtained $k_{r e a l, i i}$ on the diagonal is calculated and substituted in each element of the diagonal (this step is only required if it assumed that all connection stiffnesses are equal)

- $\quad$ The resulting matrix is used in the coupling problem to evaluate $R U_{\text {coup }}^{I I}$

- $\quad U^{I I}$ is evaluated by decoupling $\mathrm{R}$ from $R U_{\text {coup }}^{I I}$

- $\quad \mathrm{R}$ and $U^{I I}$ are inserted back in Eq.14 and the procedure is repeated to average out the noise 
A scheme of the explained procedure is shown in Fig. 15.

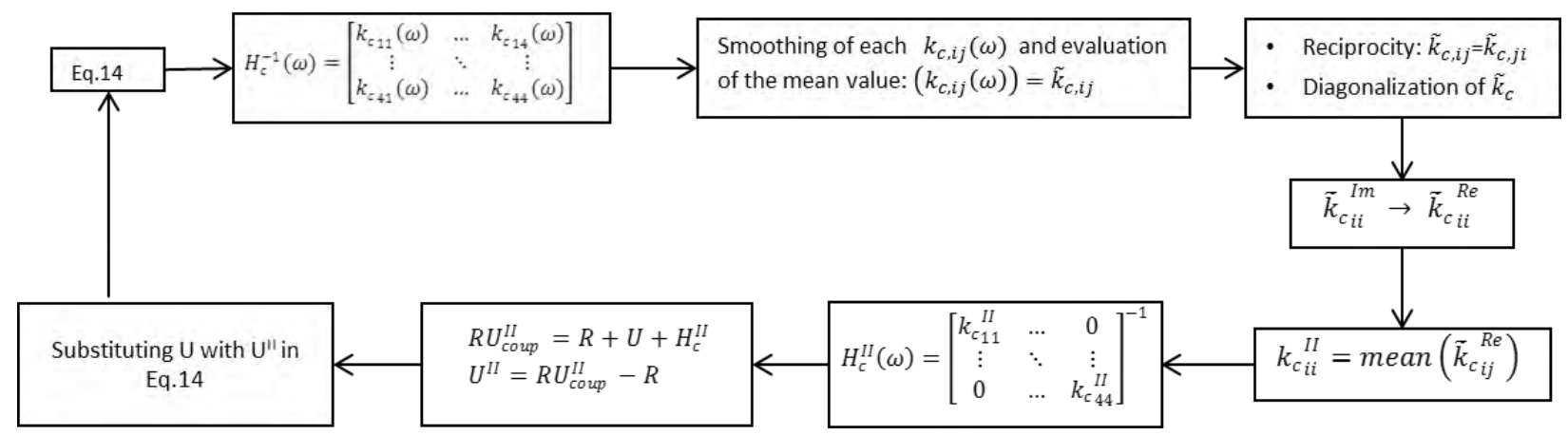

Fig. 15: Scheme of the FCU-F method for noisy data.

In the considered system, to still maintain the link with the reference model presented in Fig.4, a relatively high initial stiffness value for the connection spring needed to be defined. As a consequence, the method only needs to apply limited modification to this value to converge to an optimal solution. In Fig. 16 and Fig. 17, the results of the performed analysis are shown: note that, by only smoothing the data, values of the obtained $k_{c}$ are not accurate and the out-of-diagonal terms have purely numerical meaning (non-uniqueness of the solution). Even if not exact, we will carry on an evaluation of these results, since our aim is to verify if this value can be applied to a modified system at time $\bar{T}$ and conquently obtain more reliable results without having to perform again a full experimental campaign. For this purpose, let us to consider the same previous modified system with an added concentrated mass at the center of the beam U. In Table 2, the results for the first and the modified system affected by different levels of noise are reported: the order of magnitude is always the same, but the amplitude is almost equal up to a certain level of noise. We can conclude that, for low levels of noise, the value obtained from experimental results should not change from $T_{0}$ to $\bar{T}$ and so the connection information can be used again for next substructuring cases. As a final validation of the approach, Fig. 18 shows the coupled FRFs using rigid and flexible connections at time $T_{0}$ (left)and $\bar{T}$ (right). In both case, in particular at higher frequencies, improved results are obtained when including flexibility at the connections. Indeed, as already mentioned, the relatively high stiffness value defined will only have a marginal effect on the lower frequencies modes compared to the assumption of rigid connection.

Table 2: Calculated values of $k_{c, i i}$ by applying the Third Method

\begin{tabular}{ccc}
\hline Noise level $[\%]$ & $\boldsymbol{K}_{c, i i}$ at time $\boldsymbol{T}_{\boldsymbol{0}}[\mathbf{N} / \mathbf{m}]$ & $\boldsymbol{K}_{c, i i}$ at time $\overline{\boldsymbol{T}}[\mathbf{N} / \mathbf{m}]$ \\
\hline $\mathbf{0 . 0 0 1}$ & $1.397 \cdot 10^{10}$ & $1.412 \cdot 10^{10}$ \\
$\mathbf{0 . 0 1}$ & $6.741 \cdot 10^{9}$ & $6.845 \cdot 10^{9}$ \\
$\mathbf{0 . 1}$ & $4.523 \cdot 10^{8}$ & $8.007 \cdot 10^{8}$ \\
\hline
\end{tabular}

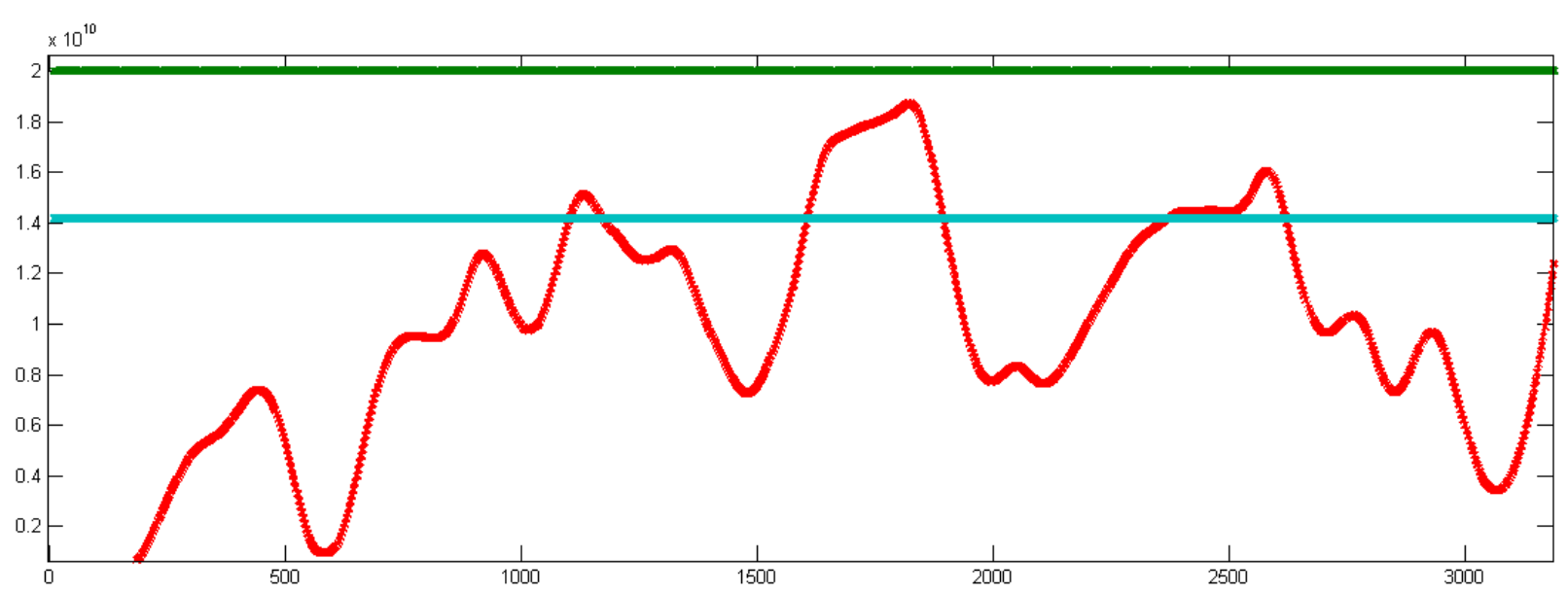

Fig. 16: Noise level: $0.001 \%$. Diagonal term of the matrix $k_{c}$ : smoothed (red), exact (green), FUC-F (cyan). 


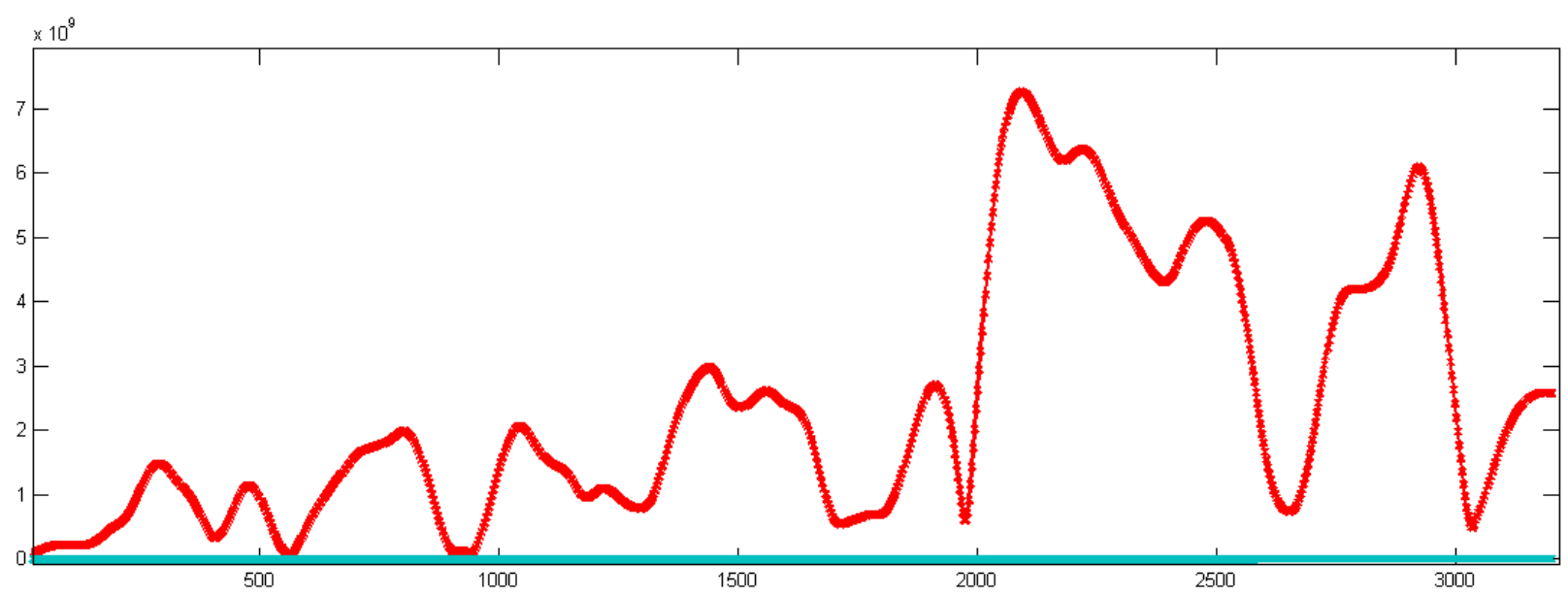

Fig. 17: Noise level: $0.001 \%$. Out-of-diagonal term of the matrix $k_{c}$ : smoothed (red), exact (green), FCU-F (cyan).
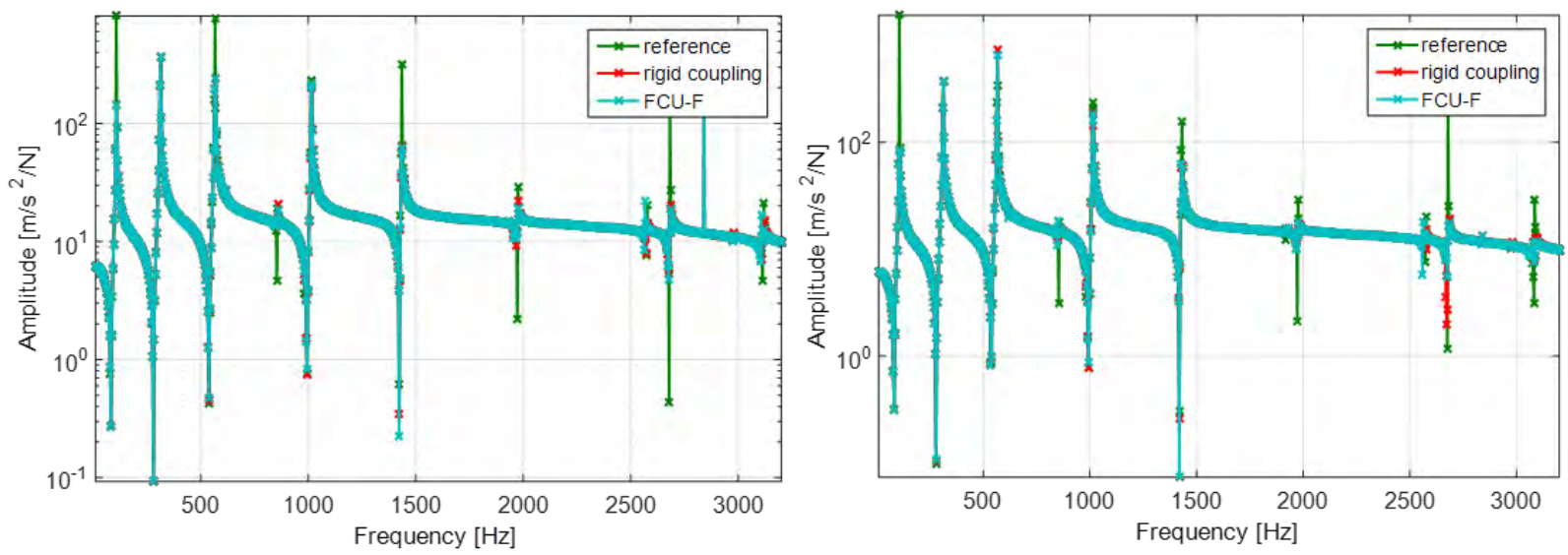

Fig. 18: Coupling results at time $T_{0}$ (left) and $\bar{T}$ (right) on DoF 5. Comparison between reference model, rigid connection assumption and flexible connection with stiffness derived using the FCU-F method

\section{CONCLUSIONS}

The paper proposes two iterative approaches for Dynamic Substructuring applications to compensate for typical experimental errors in measuring and/or modelling the connections. Sometimes, it is required to change one of the subsystem of the examined structure, thus one have to study how the dynamic behavior of the latter will be affected. Experimentally, by performing the coupling of two substructures one can observe some shift in frequencies with respect to the reference assembled RU. Here, two methods were proposed to find the connection uncertainties in the coupled system RU, by a comparison with the reference one. Then this information, associated to the type of connection, can be used again in following DS applications on the same system RU. The comparison between the two dynamic models find a useful way to compensate this frequency errors in term of mass and stiffness at the connection area. Of course the local modified properties cannot compensate in no case the lack of information about RDoFs. In fact at first the connection has to be reproduce as well. The analyzed system is composed by two beams and some simulations were performed by changing the subsystem U. The FCU-R method shows good results in the reported application: in fact the obtained mass connection error for the structure at the time $T_{0}$, led to the same percentage frequency difference in the analyzed range, also after changing U. Same considerations can be done for the application of the FCU-F method.

The disadvantage of these studies is that one should know at first the experimental (and also numerical, for the FCUR) dynamic behavior of both substructures and of the assembled RU. Anyway, after this preliminary study, the connection error could be used again in other substructuring applications (by considering the same type of 
connection). Future developments will focus on the experimental validation of the proposed methodologies, with particular attention in the applicability of the methods on different types of connections.

\section{REFERENCES}

[1] Manzato, S., Napoli, C., Coppotelli, G., Fregolent, A., D’Ambrogio, W., Peeters, B., "Experimental Coupling and Decoupling of Engineering Structures using Frequency-Based Substructuring", Dynamics of Coupled Structures, Conference Proceedings of the Society for Experimental Mechanics Series, 4, 2016, pp. 447-461.

[2] De Klerk, D., Rixen, D., Voormeeren, S. N., "General framework for dynamic substructuring: history, review and classification of techniques", AIAA Journal 46(5), 2008, pp. 1169-1181.

[3] D'Ambrogio, W., Sestieri, A., "A unified approach to substructuring and structural modification problems", Shock and Vibration, 11(3), 2004, pp. 295-309.

[4] Heylen, W., Lammens, S., Sas, P., "Modal Analysis Theory and Testing”, A.5, 2007, pp. 10-20.

[5] D'Ambrogio, W., Fregolent, A., "The role of interface DoFs in decoupling of substructures based on the dual domain decomposition", Mechanical Systems and Signal Processing, 24(7), 2010, pp. 2035-2048.

[6] Rixen, D.J., "How measurement inaccuracies induce spurious peaks in Frequency Based Substructuring", Proceedings of the International Modal Analysis Conference (IMAC), Jacksonville, USA, 2010.

[7] De Klerk, D., Rixen, D. J., Voormeeren, S. N., Pasteuning, F., "Solving the RDoF Problem in Experimental Dynamic Substructuring", Proceedings of the XXVI International Modal Analysis Conference (IMAC), Orlando, FL, 2008.

[8] Nicgorski, D. and Avitabile, P., "Experimental issues related to frequency response measurements for frequency-based substructuring", Mechanical Systems and Signal Processing, 24, 2010, pp. 1324-1337.

[9] Manzato, S., Risaliti, E., Napoli, C., Tamarozzi, T., Peeters, B., "A review of Frequency-based Substructuring methods and their applicability to Eengineering structures", Proceedings of the International Conference on Structural Engineering Dynamics (ICEDyn) 2015, Lagos, Portucal, 2015.

[10] D'Ambrogio, W., Fregolent, A., "Substructure decoupling without using rotational DoFs: Fact or fiction?", Mechanical Systems and Signal Processing, 72-73, 2016, pp. 499-512.

[11] De Klerk, D., Rixen, D., De Jong, J., "The Frequency Based Substructuring (FBS) Method reformulated according to the Dual Domain Decomposition Method", Proceedings of the International Modal Analysis Conference (IMAC), St. Louise, MO, 2006.

[12] Grafe, H., "Model Updating of Large Structural Dynamics Models Using Measured Response Functions", University of London, 1998.

[13] Arras, M., Coppotelli, G., "Finite-Element Structural Updating Using Frequency Response Functions", Journal of Aircraft, 52(5), 2015, pp. 1454-1468.

[14] Barten, E., Van Der Seijs, M.V., De Klerk, D., "A Complex Power approach to characterise Joints in Experimental Dynamic Substructuring", Dynamics of Coupled Structures, Conference Proceedings of the Society for Experimental Mechanics Series, 1, 2014, pp. 281-296. 